Article

\title{
Tumour Cell Labelling by Magnetic Nanoparticles with Determination of Intracellular Iron Content and Spatial Distribution of the Intracellular Iron
}

\section{Zhigang Wang * and Alfred Cuschieri *}

Institute for Medical Science and Technology, University of Dundee, Dundee DD2 1FD, UK

* Authors to whom correspondence should be addressed; E-Mails: z.z.wang@dundee.ac.uk (Z.W.); a.cuschieri@dundee.ac.uk (A.C.); Tel.: +44-1382-381-030 (Z.W.); +44-1382-381-009 (A.C.); Fax: +44-1382-386-588 (Z.W. \& A.C.).

Received: 1 March 2013; in revised form: 26 March 2013 / Accepted: 2 April 2013 /

Published: 26 April 2013

\begin{abstract}
Magnetically labelled cells are used for in vivo cell tracking by MRI, used for the clinical translation of cell-base therapies. Studies involving magnetic labelled cells may include separation of labelled cells, targeted delivery and controlled release of drugs, contrast enhanced MRI and magnetic hyperthermia for the in situ ablation of tumours. Dextran-coated super-paramagnetic iron oxide (SPIO) ferumoxides are used clinically as an MR contrast agents primarily for hepatic imaging. The material is also widely used for in vitro cell labelling, as are other SPIO-based particles. Our results on the uptake by human cancer cell lines of ferumoxides indicate that electroporation in the presence of protamine sulphate (PS) results in rapid high uptake of SPIO nanoparticles (SPIONs) by parenchymal tumour cells without significant impairment of cell viability. Quantitative determination of cellular iron uptake performed by colorimetric assay is in agreement with data from the literature. These results on intracellular iron content together with the intracellular distribution of SPIONs by magnetic force microscopy (MFM) following in vitro uptake by parenchymal tumour cells confirm the potential of this technique for clinical tumour cell detection and destruction.
\end{abstract}

Keywords: magnetic nanoparticles; superparamagnetic; SPIONs; nanomedicine; magnetic cell labelling; magnetic force microscopy 


\section{Introduction}

All materials are magnetic to some extent, with their response depending on their atomic structure and temperature. They can be conveniently classified in terms of their volumetric magnetic susceptibility, $\chi$, where $M=\chi H$ describes the (volumetric) magnetization $M$ induced in a material by magnetic field (strength), $H$, with the material (relative) permeability, $\mu_{\mathrm{r}}$, being defined as $\mu_{\mathrm{r}}=(1+\chi)$. In SI units, $\chi$ is dimensionless, and both $M$ and $H$ are expressed in $\mathrm{Am}^{-1}$. Most materials display little magnetism and, even then, only in the presence of an applied field; these are classified either as paramagnets, for which $\chi$ falls in the range $10^{-6}$ to $10^{-1}$, or diamagnets, with $\chi$ being in the range $-10^{-6}$ to $-10^{-3}$. However, some materials exhibit ordered magnetic states and are magnetic even without an applied field. These are classified as ferromagnets, ferrimagnets and antiferromagnets, where the prefix refers to the nature of the coupling interaction between the electrons within the material. This coupling can give rise to large spontaneous magnetizations; in ferromagnets, $M$ is typically $10^{4}$ times larger than would otherwise be the case.

The magnetic properties in ferromagnetic materials are the result of aligned unpaired electron spins. For these materials, magnetization is evident even in the absence of an external field. The transition between two magnetic domains (so-called Weiss domains) is referred to as a Bloch wall. At the nanometre scale (of the order of tens of nanometres or less, e.g., $\sim 14 \mathrm{~nm}$ ), the formation of Bloch walls becomes thermodynamically unfavourable, leading to the formation of single domain crystals, which are classified as superparamagnetic. The term superparamagnetic refers to the characteristic strong paramagnetic nature of the particles at this scale. Paramagnetic materials are distinguished by the tendency of their atomic magnetic dipoles to align with an external magnetic field, their small positive magnetic susceptibility (i.e., ability to strengthen the field they are in) and their random orientation in the absence of a magnetic field (due to Brownian fluctuations). Superparamagnetic iron oxide (SPIO) nanoparticles have much larger susceptibilities (compared with strictly paramagnetic materials), as the entire crystal aligns with the applied field, due to its single crystal nature [1].

Magnetic nanoparticles offer a lot of attractive possibilities in biomedicine [2,3], and magnetic cell labelling is considered essential for the translation of cell-base therapies from the laboratory to clinical studies. Examples include magnetic separation of labelled cells and biological entities [4], targeted delivery and controlled release of drugs [5,6], magnetic hyperthermia for the in situ ablation of tumours [7] and contrast enhanced MRI [8-10]. Molecular and cellular magnetic resonance (MR) imaging is a rapidly growing field that aims to visualize targeted macromolecules or cells in living organisms by the use of superparamagnetic iron oxide (SPIO) nanoparticles (SPIONs) [1,11]. MR cell tracking, with its excellent spatial resolution, can be used as a non-invasive tool to provide unique information on the dynamics of cell movements in vivo. It is likely that MR cell tracking will be used in the future to monitor (stem) cell therapy in patients [12]. All these approaches require magnetic labelling of cells, as well as methods for analysis and evaluation of cell labelling [13,14]. Due to their biocompatibility and strong effects on $\mathrm{T} 2 *$ relaxation, iron oxide nanoparticles are now the MR contrast agent of choice for cell labelling [15], and several methods have been developed to incorporate sufficient quantities of iron oxide nanoparticles into cells [16,17].

A variety of cells have the natural ability to internalize exogenous material by a process known as endocytosis. The size of material and the rate at which it is internalized is dependent on the specific 
trans-membrane mechanism involved in the passage through the cell membrane. While specific cellular labelling by targeting specific binding sites on the surface of cells is possible, using SPIO nanoparticles coated with antibodies or other biological macromolecules, such as hormones or folic acid [18], our studies focused on non-specific cellular labelling. The majority of nonspecific cellular labelling has to date involved macrophages, as these cells are capable of efficient phagocytosis of exogenous/foreign particles, including micrometre-sized iron oxide particles [19]. In more general cases, the iron cellular uptake is not sufficient without the use of transfection agents or linking nanoparticles to the highly cationic HIV TAT peptide [20] or by using other physical means, such as magnetofection [21], electroporation [22] or sonoporation [23]. For example, cells have been successfully magnetically labelled by simply adding magneto-dendrimers, synthesized dendrimer encapsulated SPIOs [24], to the culture medium at concentrations of up to $25 \mu \mathrm{g}$ iron/mL and incubation periods of one to two days.

For cell labelling application, magnetic nanoparticles have surface modification, usually by coating with biocompatible molecules, e.g., dextran, polyvinyl alcohol (PVA) and phospholipids. As well as providing a link between the particle and the target site on a cell or molecule, coating has the advantage of increasing the colloidal stability of the magnetic fluid. Ferumoxide (Feridex; Berlex, Wayne, NJ, USA) is a dextran-coated iron oxide [25], with particles sized between 80 and $120 \mathrm{~nm}$. Ferumoxide is an FDA approved hepatic contrast agent, because the nanoparticles are spontaneously internalized by phagocytes (Kupffer cells). On post-contrast MRI scans, the phagocyte-rich liver turns dark, while the tumour, lacking macrophages, remains iso-intense (white). In our studies, for magnetic pre-labelling of non-phagocytic cells and cellular MRI, Ferumoxides have been combined with other commercially available transfection agents (TAs), such as poly-L-lysine (PLL) (Sigma, St. Louis, MO, USA) and Lipofectamine Plus (Invitrogen Life Technologies, Gaithersburg, MD, USA) [26].

We investigated several protocols for magnetic labelling of human cancer cells with ferumoxides [25] utilizing transfection agents, including poly-L-lysine (PLL), poly-L-ornithine (PLO) and protamine sulphate (PS), and applying electroporation [27]. In this report, we describe a quantitative assessment of magnetically labelled cells using conventional iron uptake measurement, as well as a magnetic force microscopy (MFM) study, which has the capability to resolve the spatial distribution of internalized magnetic nanoparticles [28-30] and can also be used to estimate the true uptake by a single cell and its resulting magnetization.

\section{Results and Discussion}

\subsection{Magnetic Labelled Cells-Efficiency and Viability}

Cells were successfully labelled by magnetic nanoparticles (IO-nPs or ferumoxides) using Prussian blue staining, as illustrated (Figure 1). The resulting cell labelling efficiency and cell viability for three labelling methods used in this study are shown in Table 1. A very high labelling efficiency (95\%) was obtained using protamine sulphate (PS) alone, while electroporation (EP) was only capable of loading cells with an efficiency of $72 \%$. When PS was added to magnetic nanoparticles solution in electroporation mixtures, the loading efficiency increased from $72 \%$ to $88 \%$. 
Figure 1. Cell labelling by electroporation and PS/electroporation: (a) stained promptly after electroporation (Cytospin staining); (b) stained after overnight culture. PS, protamine sulphate; EP, electroporation; IO-nPs, ferumoxides.

(a)

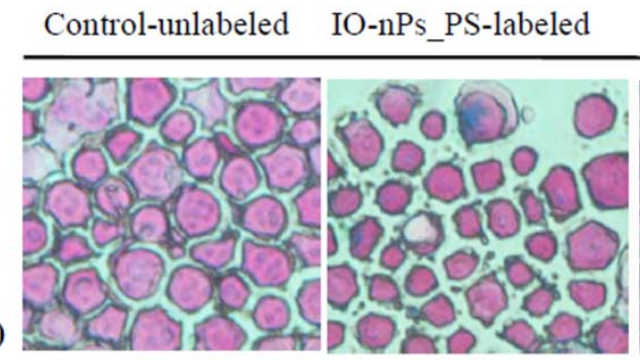

IO-nPs_EP-labeled IO-nPs_PS/EP-labeled

(b)

Labeling efficiency:

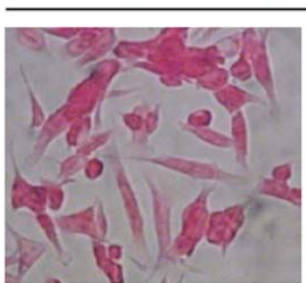

Labeling efficiency:
$<5 \%$

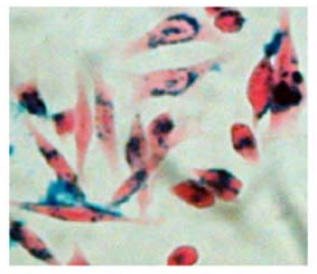

$95 \%$

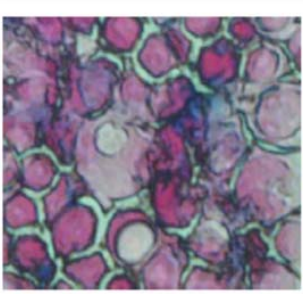

$72 \%$

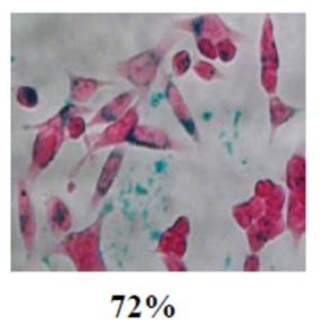

$88 \%$

(N/A)

Table 1. Comparison of cell labelling by transfection, electroporation and PS/electroporation (PS, protamine sulphate; EP, electroporation; IO-nPs, ferumoxides).

\begin{tabular}{cccc}
\hline Labelling method & PS & EP & PS/EP \\
\hline Final concentration of IO-nPs $(\mu \mathrm{g} / \mathrm{mL})$ & 30 & 100 & 100 \\
Final concentration of PS $(\mu \mathrm{g} / \mathrm{mL})$ & 3.0 & - & 3.0 \\
Duration of procedure & $14-16 \mathrm{~h}$ & $30 \mathrm{~min}$ & $30 \mathrm{~min}$ \\
Labelling efficiency $(\%)$ & 95 & 72 & 88 \\
Cell viability (A 375M) & $98.73 \pm 5.56$ & $73.21 \pm 7.21$ & $89.34 \pm 3.56$ \\
\hline
\end{tabular}

With incubation of cells with PS, nanoparticle solution with PS did not significantly affect cell proliferation, but electroporation significantly decreased cell viability compared with controls, while combination (electroporation + PS) labelling significantly improved cell viability (Table 1). This combination labelling was also fast (i.e., $30 \mathrm{~min}$ ), obviating the need for prolonged incubation by transfection agents, i.e., overnight (see Table 1 for comparison).

\subsection{Assessment Cellular Iron Uptake and Spatial Distribution}

The standard optical density (OD) curve in a 96-well plate using the Quantichrom iron assay was plotted (Figure 2). This had a slope of 0.0005 with $R^{2}=0.9996$. Using Equation (3) (described in Experimental Section), the amount of intracellular iron was estimated to be $3.773 \pm 0.348(n=4)$ for the A375M cell line and $4.115 \pm 0.564(n=4)$ for the MCF7 cell line (Table 2$)$. 
Figure 2. Standard curve in 96-well plate assay.

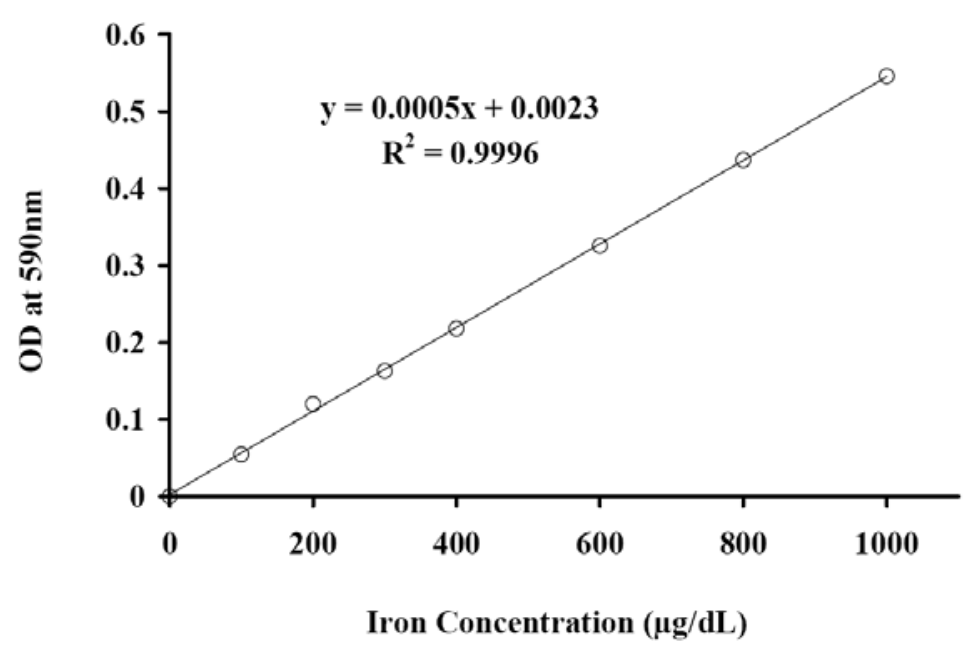

Table 2. Quantitative cellular iron uptake: labelled with IO-nPs (ferumoxides) using PS/EP and measured by the Quantichrom iron assay (PS, protamine sulphate; EP, electroporation).

\begin{tabular}{ccc}
\hline Iron uptake & Labelled (pg/cell) & Control (pg/cell) \\
\hline A375M (melanoma) & $3.773 \pm 0.348(n=4)$ & $0.075 \pm 0.130(n=4)$ \\
MCF7 (breast) & $4.115 \pm 0.564(n=4)$ & $0.179 \pm 0.229(n=4)$ \\
\hline
\end{tabular}

The spatial distribution of SPIOs following cellular uptake was demonstrated by stained optical images (Figure 1) and can be observed more closely in the accompanying MFM images (Figures 3 and 4). Cells can be observed in their morphological images in Figure $3 \mathrm{a}$ for a labelled cell and for an unlabelled (control) cell in Figure 3c. The uptake of SPIOs uptake is clearly shown in the phase (retrace) image (Figure 3b) for the labelled cell, while no such phase shift was detected in the control cell (Figure 3d).

Figure 3. Magnetic force microscopy (MFM) images showing nanoparticles' uptake and spatial distribution within single cells: $(\mathbf{a}-\mathbf{b})$ a labelled cell with morphological images in (a) and SPIOs uptake and a spatial distribution in phase (retrace) image (b); (c-d) an unlabelled cell for control with morphological images in (c), and no such phase shift detected in (d).

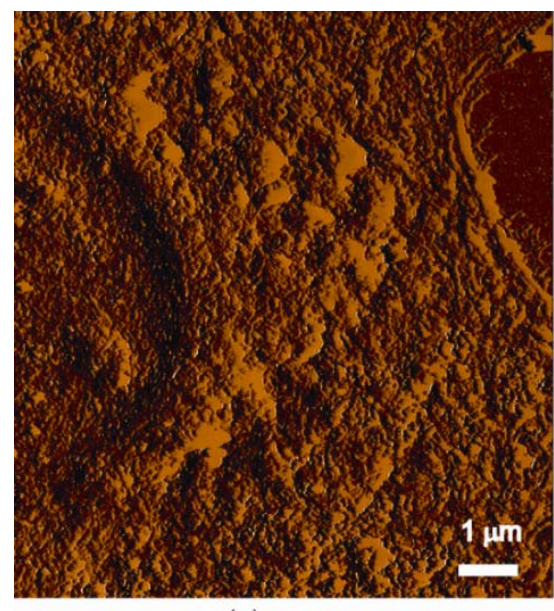

(a)

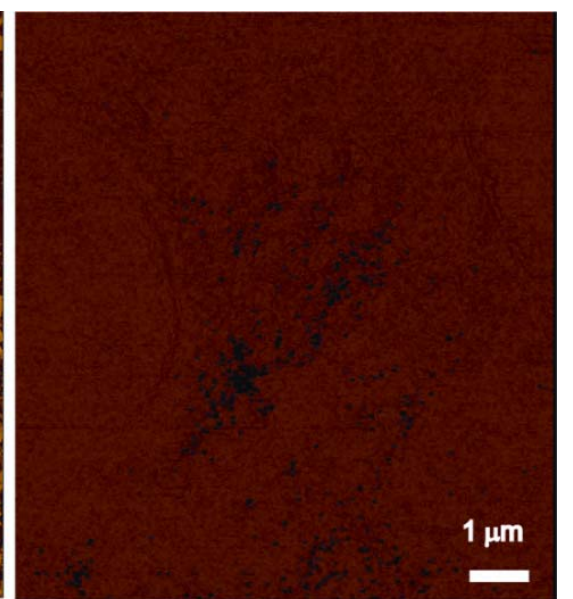

(b) 
Figure 3. Cont.

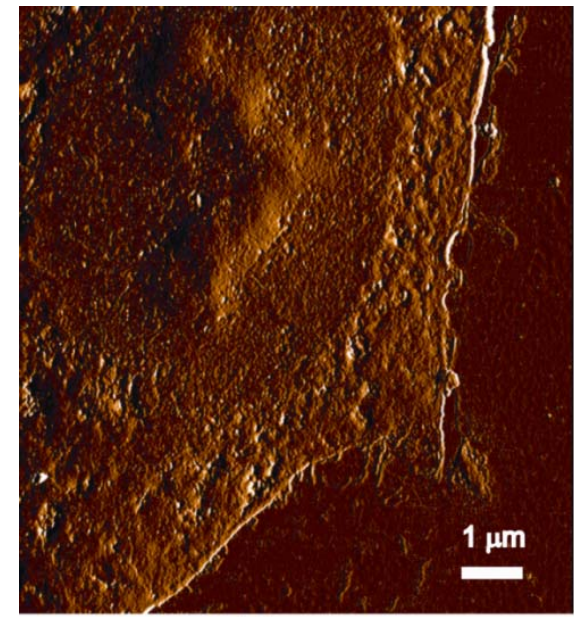

(c)

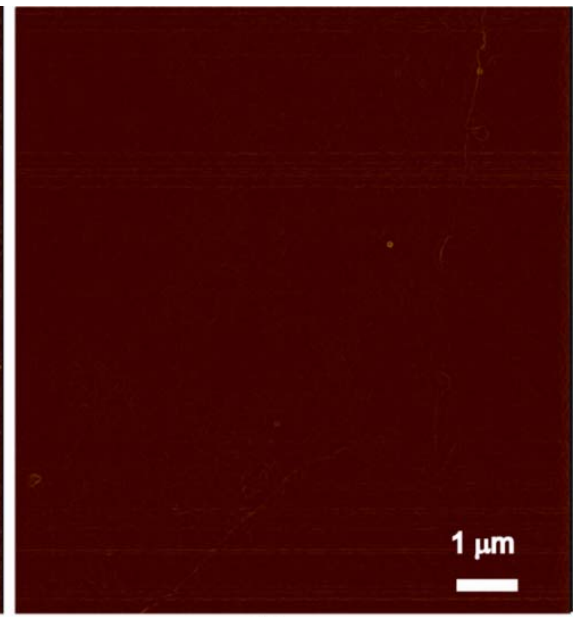

(d)

Figure 4. MFM images of a single cell: (a) 3D morphological image of the cell; (b) phase image in retrace mode (lift height of $100 \mathrm{~nm}$ from cell surface) showing SPIOs uptake and spatial distribution.

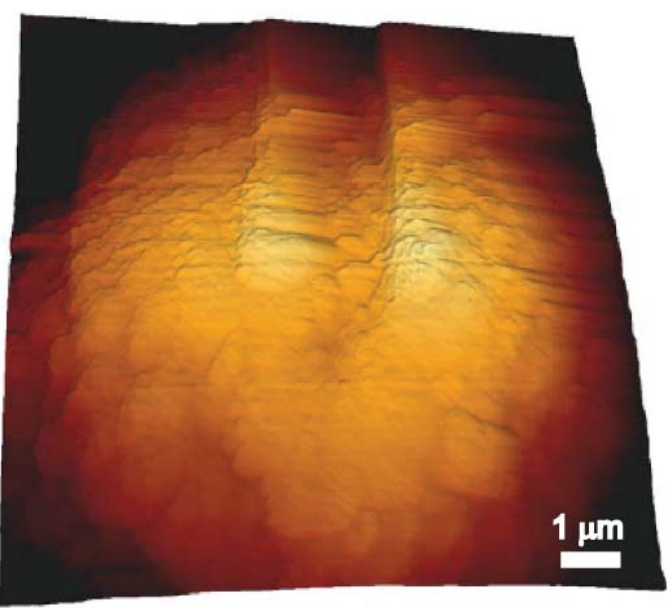

(a)

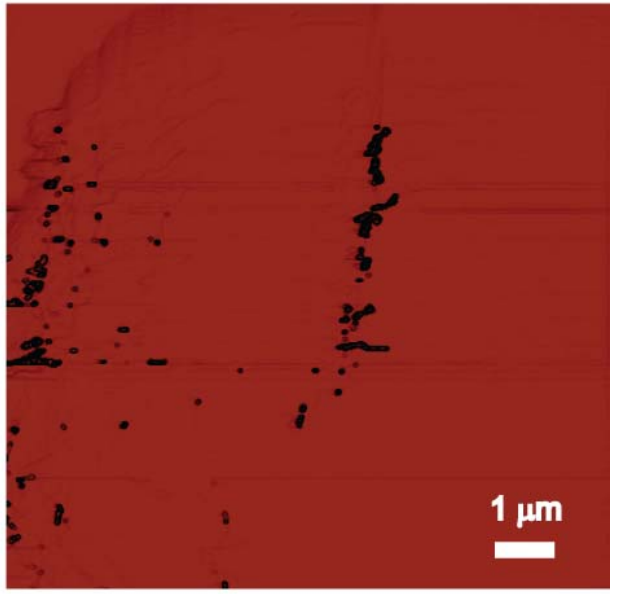

(b)

SPIOs uptake by a single cell was observed using MFM (Figure 4), the quantitative iron uptake by the cell being estimated by Equation (5) (described in Experimental Section) at around 1.9 pg. The double-layer model provides an approximate iron uptake of $3.8 \mathrm{pg}$ per cell.

\subsection{Discussion}

It is important to develop simple, accurate and low-cost methods for the determination of SPIOs iron for both clinical research and industrial work. The simplest iron determination method is the spectrophotometric one, which is based on protein precipitation, reduction of $\mathrm{Fe}^{3+}$ and formation of a coloured complex of $\mathrm{Fe}^{2+}$ with a chromogen [31]. The intensity of the colour, measured at $590 \mathrm{~nm}$ by spectrophotometry, colorimeter or a microplate reader being directly proportional to the iron concentration in the sample. 
Iron content within in vitro labelled cells was evaluated using the Quantichrom iron assay. Wu et al. [32] reported that when $50 \mu \mathrm{L}$ of samples containing $10^{6}$ cells were mixed with $200 \mu \mathrm{L}$ Quantichrom Working Reagent in a 96-well plate (in triplicate) and incubated at room temperature overnight, the iron content per cell increased from $0.32 \mathrm{pg}$ (protamine only) to $0.84 \mathrm{pg}$ (ferumoxides) and $26.0 \mathrm{pg}$ (Fe-Pro complex) $2 \mathrm{~h}$ after cell labelling.

The ferrozine-based assay is acknowledged as the most reliable method for colorimetric quantification of iron [22,24,26]. Ferrozine is an iron-chelating agent that forms a complex with ferrous iron $\left(\mathrm{Fe}^{2+}\right)$ and exhibits characteristic UV-Vis absorption at $562 \mathrm{~nm}$. The amount of intracellular iron as determined by the ferrozine-based colorimetric assay ranges between 1 and $5 \mathrm{pg} \mathrm{Fe} / \mathrm{cell}$, depending on the electroporation pulse conditions [22]. A more detailed description of colorimetric ferrozine-based assay can be found in [33]. This sensitive assay permits the quantification of iron in cultured cells in amounts ranging between 0.2 and $30 \mathrm{nmol}$. More importantly, estimates of cellular iron content obtained with the ferrozine-based assay are similar to those determined by the more expensive atomic absorption spectroscopy, MR relaxometry and radioactive detection, described below.

Atomic spectrometry: Inductively coupled plasma-atomic emission spectrometry (ICP-AES), also referred to as inductively coupled plasma optical emission spectrometry (ICP-OES), is a well-established technique for elemental analysis. For cellular iron uptake determination, the technique is based on the measurement of the emitted light of excited iron atoms. In the quantitative determination of intracellular iron uptake by human lung carcinoma cells (CLL-185) [8] by ICP-AES revealed a dose-dependent increase of iron oxide uptake $(1.69 \mu \mathrm{g} \pm 0.11 \mathrm{Fe}$ per 100,000 cells at $1.0 \mathrm{mg} \mathrm{Fe} / \mathrm{mL}$ vs. $0.08 \mu \mathrm{g} \pm 0.01 \mathrm{Fe}$ per 100,000 cells at $0.01 \mathrm{mg} \mathrm{Fe} / \mathrm{mL} ; p<0.05$ ) (Table 3). Inductively coupled plasma-mass spectrometry (ICP-MS) is an analytical technique that measures the mass-to-charge ratio of charged particles. Different chemicals have different masses, and this fact is used in mass spectrometry to identify chemicals present in a sample. The cellular uptake of micro-sized iron oxide particles analysed [19] by ICP-MS was reported to be $35 \mathrm{pg}$ of Fe/cell (Table 3), which is consistent with results obtained by microscopy. Another clinically used paramagnetic contrast agents (apart from iron oxide based particles) is gadolinium (Gd)-based chelates, and the intracellular concentration of Gd per cell can also be measured using ICP-MS [34].

Magnetic resonance (MR) relaxometry: MR relaxometric methods are also used to measure the concentration of iron. They are based on the linear relationship between iron content and MR relaxation rates of $1 / \mathrm{T} 1$ or 1/T2 [24,26]. By using either custom designed equipment or commercially available MRI scanners, T1 and T2 relaxation rates obtained from samples are compared with the known iron concentration in serial dilutions of iron solution that is used for generating standard calibration curve. Bulte et al. [24] estimated cellular uptakes of $9.3 \pm 4.3$ and $13.6 \pm 5.5 \mathrm{pg}$ iron/cell for the CG-4 (rat oligodendrocyte progenitor) and HeLa (human cervix carcinoma) cells, respectively. This was in agreement with the corresponding values obtained using the Ferrozine assay, which was $8.5 \pm 2.0$ and $13.6 \pm 2.9$, respectively (Table 3 ). 
Table 3. Reported cellular uptake rates of iron.

\begin{tabular}{ccccc}
\hline Measurement & Uptake (pg Fe/cell) & Cells $^{\#}$ & Magnetic particles \& labelling * & References \\
\hline ICP-AES & $16.9 \pm 1.1$ & CLL-185 & SPIOs $(1 \mathrm{mg} / \mathrm{mL})$ and lipofection & {$[8]$} \\
ICP-AES & $0.8 \pm 0.1$ & CLL-185 & SPIOs $(10 \mu \mathrm{g} / \mathrm{mL})$ and lipofection & {$[8]$} \\
ICP-MS & 35 & B16F10 & MPIO beads and macrophages & {$[19]$} \\
MR relaxometry & $9.3 \pm 4.3$ & CG-4 & SPIOs $(1-25 \mu \mathrm{g} / \mathrm{mL})$ and dendrimers & {$[24]$} \\
Ferrozine assay & $8.5 \pm 2.0$ & CG-4 & SPIOs $(1-25 \mu \mathrm{g} / \mathrm{mL})$ and dendrimers & {$[24]$} \\
MR relaxometry & $13.6 \pm 5.5$ & HeLa & SPIOs $(1-25 \mu \mathrm{g} / \mathrm{mL})$ and dendrimers & {$[24]$} \\
Ferrozine assay & $13.6 \pm 2.9$ & HeLa & SPIOs $(1-25 \mu \mathrm{g} / \mathrm{mL})$ and dendrimers & {$[24]$} \\
Relaxometry/Ferrozine & $3.8 \pm 1.2$ & CG-4 & Ferumoxides and PLL $(25 \mu \mathrm{g} / \mathrm{mL})$ & {$[26]$} \\
Gamma counter and ${ }^{111}$ In & 10 to 30 & CD34 & CLIO-Tat peptides $(100 \mu \mathrm{g} / \mathrm{mL})$ & {$[20]$} \\
Ferrozine assay & 1 to 5 & NSC $(C 17.2)$ & Ferumoxides $(2 \mathrm{mg} / \mathrm{mL})$ and EP & {$[22]$} \\
Quantichrom assay & 26.0 & Leukocytes & Ferumoxides $(50 \mu \mathrm{g} / \mathrm{mL})$ and PS & {$[32]$} \\
\hline \# Cell lines used in in vitro measurement-B16F10: melanoma; CLL-185: human lung carcinoma cells; CG-4: rat \\
oligodendrocyte progenitor; HeLa: human cervix carcinoma; CD34 $4^{+}$human hematopoietic cells; MSC: mesenchymal \\
stem cells; NSC (C17.2): neural stem cells. * Labelling method-EP: electroporation; PS: protamine sulphate; ICP-AES, \\
inductively coupled plasma-atomic emission spectrometry; ICP-MS, inductively coupled plasma-mass spectrometry.
\end{tabular}

Radioactive detection: In this technique, Dextran-coated SPIO are modified with diethylenetriamine penta-acetic acid (DTPA) for isotope labelling, i.e., using a radiotracer $\left({ }^{111} \mathrm{In}\right)$. The radiolabeled cross-linked iron oxide (CLIO)-TAT particles, counted with a gamma counter, result in labelling efficiencies ranging [20] from 10 to $30 \mathrm{pg}$ of superparamagnetic iron per cell (Table 3). Our in vitro study suggests that electroporation in the presence of protamine sulphate (PS) as a transfection agent can provide an effective and fast technique for labelling various types of cells with magnetic nanoparticles and provides a method that can be used clinically. Our quantitative results obtained by the Quantichrom iron assay (3.8 to $4.1 \mathrm{pg}$ per cell) are in good agreement with the values in the published literature (i.e., from 0.8 to $35 \mathrm{pg} /$ cell, Table 3 ), depending on cell types, SPIOs size and concentration, surface modification, incubation time, etc. The MFM imaging data demonstrate the spatial distribution of internalized IO-nPs. Furthermore, with the simplified geometrical model, the MFM-based single cell imaging assessment of IO-nPs' uptake can provide additional quantitative information on the individual magnetized cell (1.9 to $3.8 \mathrm{pg}$ ). More advanced modelling and simulation would provide more accurate quantitative information of single-cell magnetization based on MFM scanned image and other imaging modality, such as TEM, to estimate SPIOs' aggregation depth inside the cell [30].

Finally, magnetofection is a relatively new transfection method and is achieved by the application of a magnetic field to superparamagnetic iron oxide particles [35]. Magnetofection exploits the magnetic forces that guide the SPIOs, associated with gene vectors, DNA plasmids, toward the target cells. The presence of these magnetic fields increases the transfection efficiency, compared to cells not exposed to the magnetic field [35-39]. Further investigation with magnetic nanoparticles coated with positively charged groups [40] for more cell labelling experiments, especially with the used of the our recently reported magnetoporation method [41] by an low-intensity external rotating magnetic field, is underway, since magnetoporation/magnetofection have potential over electroporation in clinical applications. 


\section{Experimental Section}

\subsection{Cell Labelling by Magnetic Nanoparticles}

We investigated different magnetic cell labelling methods using magnetic nanoparticles (SPIOs or IO-nPs), including transfection agents and electroporation. Our aim was to identify an effective and fast technique for potential clinical use. The work was based on the hypothesis that SPIOs, coated and mixed as complexes with cationic transfection agents, can be more effectively aligned to cell membranes, as these are slightly negatively charged, thereby achieving more efficient labelling through trans-membrane passage by electropermeabilization and other mechanisms, including natural endocytosis. Several human cancer cell lines (A375M, DLD1, MCF7, SW480 and U2OS) were used for the labelling studies.

\subsubsection{Labelling with Transfection Agent (PS)}

The transfection agent, protamine sulphate (PS), at varying concentrations was mixed with IO-nPs $(60 \mu \mathrm{g} / \mathrm{mL})$ in culture media for $15 \mathrm{~min}$ at room temperature with intermittent hand shakings. The culture medium containing the transfection agent-IO-nPs complexes were added to the cell cultures such that the final concentration of IO-nPs was $30 \mu \mathrm{g} / \mathrm{mL}$, and the final concentrations of PS ranged between $1.0-5.0 \mu \mathrm{g} / \mathrm{mL}$ in cell viability experiments and $3.0 \mu \mathrm{g} / \mathrm{mL}$ for other experiments. Cells were then incubated overnight, for approximately $16-18 \mathrm{~h}$.

\subsubsection{Labelling with Electroporation}

Electroporation was performed with a Nucleofector Device II from Amaxa Biosystems GmbH (Cologne, Germany), according to the manufacturer's instructions. Cells suspended in Nucleofector Solutions were mixed with IO-nPs media before transfer to electroporation cuvettes. Specific intensities and lengths of electric pulse were selected and used to obtain optimal labelling. Control experiments were performed by processing cells in the same way, but in the absence of IO-nPs. After electroporation, cell suspensions were diluted with pre-warmed complete DMEM and transferred to culture plates. The final concentration of IO-nPs in culture medium was $100 \mu \mathrm{g} / \mathrm{mL}$. Cells were incubated overnight or immediately assessed for instant labelling or cell viability.

\subsubsection{Labelling with Electroporation in the Presence of PS}

This was carried out by the same procedure and addition of PS-IO-nPs complexes to the pre-warmed medium used to dilute the electroporation mixtures. In order to maintain comparable conditions, the final concentrations were kept at $3 \mu \mathrm{g} / \mathrm{mL}$ and $100 \mu \mathrm{g} / \mathrm{mL}$ for PS and IO-nPs, respectively.

\subsection{Assessment of Magnetically Labelled Cells}

\subsubsection{Cell Viability by MTS Assay}

The MTS Cell Titre 96 AQueous One Solution Cell Proliferation Assay (Promega, Southampton, UK) was used to determine cell viability in control and treated cell studies. Cells were seeded at 
densities of 5000-10,000 cells/well in 96-well plates and were either assayed at $30 \mathrm{~min}$ (after electroporation) or left to adhere for 16-18 h for transfection treatments before MTS assay. The MTS assay values (absorbance at $490 \mathrm{~nm}$ ) were expressed as the percentage of that of the corresponding control cells.

\subsubsection{Prussian Blue Staining for Assessing Labelling Efficiency}

Cells grown on glass coverslips were washed with PBS to remove excess of IO-nPs and fixed with $4 \%$ para-formaldehyde for $30 \mathrm{~min}$. They were then washed with ddH2O and incubated for $30 \mathrm{~min}$ with $2.5 \%$ potassium ferrocyanide in $2.5 \% \mathrm{HCl}$ following counterstaining with nuclear fast red for $5 \mathrm{~min}$. The samples were examined using a Zeiss microscope Axiovert 200 (Zeiss, Oberkochen, Germany) at $\times 40$ and $\times 63$ magnification and Axiovision 4.6 software. Cells were considered Prussian Blue positive if intra-cytoplasmic blue granules could be identified. The efficiency of cell labelling was determined by manual counting of Prussian Blue-positive and -negative cells. The percentage of labelled cells was determined from the average of 5 to 10 fields $(\times 40)$.

\subsubsection{Quantitative Colorimetric Iron Assay}

The Quantichrom iron assay (BioAssay Systems, Hayward, CA, USA), a quantitative colorimetric iron determination at $590 \mathrm{~nm}$, was used for studying cellular iron uptake. In accordance with the manufacturer's protocol, $50 \mu \mathrm{L}$ of standards or samples containing $10^{6}$ cells were mixed with $200 \mu \mathrm{L}$ Quantichrom Working Reagent in a 96-well plate and incubated at room temperature overnight [32]. In this system, the optical density (OD) at $590 \mathrm{~nm}$ measured by a microplate reader is directly proportional to the iron concentration in the sample. The OD against standard iron concentrations were plotted by subtracting blank (water) OD from the standard OD values, and the slope of the data plot then determined using liner regression fitting (Slope $=0.0005$, see Figure 3 ). The iron concentration ( $\mathrm{IC}_{\text {sample }}$ in $\mu \mathrm{g} / \mathrm{dL}$ ) of the labelled sample can be calculated as:

$$
\mathrm{IC}_{\text {sample }}=\frac{\mathrm{OD}_{\text {sample }}-\mathrm{OD}_{\text {blank }}}{\text { Slope }}
$$

where $\mathrm{OD}_{\text {sample }}$ and $\mathrm{OD}_{\text {blank }}$ are $\mathrm{OD}_{590 \mathrm{~nm}}$ values of the labelled cell suspension sample and the blank water sample. Given cell concentration $\left(\mathrm{CC}_{\text {sample }}\right)$ in the labelled cell suspension sample of $N(\mathrm{cell} / \mathrm{dL})$ $\left(N=10^{6}\right.$ cells $/(50 \mu \mathrm{L}+200 \mu \mathrm{L})$ in this assay), averaged iron uptake per cell (iron uptake in $\mathrm{pg} / \mathrm{cell}$ ) can be obtained from:

$$
\text { IronUptake }=\frac{\mathrm{IC}_{\text {sample }}}{\mathrm{CC}_{\text {sample }}}=\frac{\mathrm{OD}_{\text {sample }}-\mathrm{OD}_{\text {blank }}}{\text { Slope }} \frac{1}{N} 10^{6}
$$

\subsection{Magnetic Force Microscopy (MFM) Observation and Assessment}

\subsubsection{Magnetic Force Microscopy (MFM) Principle}

MFM is a special mode of operation of the non-contact scanning atomic force microscope (AFM), with high $(\sim 25-50 \mathrm{~nm})$ spatial resolution [28,29]. The principle of MFM is to measure the change of 
the interaction force $\left(F_{\mathrm{m}}\right)$ between a magnetized probe (of strength $H$ ) and the local stray magnetic field (induction), $B$, from the sample as the probe is scanned across the surface in dynamic (non-contact) mode by :

$$
F_{m}=(m \cdot \nabla) B
$$

where $m=M V$ is the magnetic moment on a volume, $V$, of the material with magnetisation of $M$ ( $M=\chi H$ and $\chi$ is material's volumetric magnetic susceptibility, which can be obtained SPIOs' material magnetization curve), and each individual atomic moments in the material contribute to its overall magnetic induction $(B)$ response as:

$$
B=\mu_{0}(H+M)
$$

where $\mu_{0}$ is the permeability of free space.

One standard method of obtaining a MFM image is to operate the AFM in close-contact mode with a magnetic cantilever that detects a force gradient, which contains information from both the surface structure and the local magnetic field. After collecting a topographic image close to the surface, the cantilever is then 'raised' some height above the surface, where the magnetic forces dominate on the reverse scan. Signals from surface topography dominate at close distances to the surface, while at greater distances from the surface, the magnetic signal dominates. Consequently, depending on the distance between the surface and the tip, normal MFM images may contain a combination of topographic and magnetic signals. Standard magnetic recording tape was used to obtain known magnetic domain pattern image for assisting selection of the MFM tip lift height (e.g., $100 \mathrm{~nm}$ in this study).

\subsubsection{MFM Scans with Image Processing for Single Cell Assessment}

IO-nPs labelled cells (SW480) were prepared, and the air dried glass slides of samples were used for MFM. Cell uptake was imaged using a JPK Nanowizard atomic-force microscope (AFM) (JPK Instruments, Berlin, Germany) on top of an Axiovert 200 inverted microscope (Carl Zeiss, Jena). MFM imaging using phase detection was performed in hover (lift) mode within intermittent contact (dynamic) mode. The lift height was $100 \mathrm{~nm}$ distance between the cantilever tip and the surface of the sample, and the MFM scan was performed at a scan rate of $1 \mathrm{~Hz}$ with a resolution of $512 \times 512$ pixels. The MFM cantilevers were made of silicon coated with cobalt-chromium alloy (Hc $=300-400$ Oe, spring constant: 1-5 N/m, MFMR-10, Nanosensors Inc., Darmstadt, Germany).

MFM scan image of SPIOs uptake with a complete single cell was then further processed by a custom-written Matlab (MathWorks, Cambridge, UK) codes for imaging analysis to obtain a total

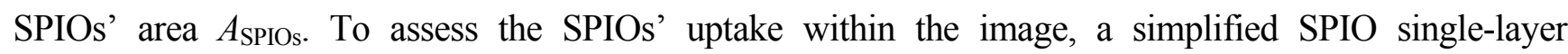
geometrical model was used to obtain the SPIOs' volume $V\left(V=A_{\text {SPIOs }} \times D_{\text {SPIO }}\right.$, where $D_{\text {SPIO }}$ is the diameter of a single SPIO particle). Given a volumetric density, $\rho$, of the used SPIOs, we could estimate the mass $M_{\mathrm{SPIOs}}$ of the uptake SPIOs by:

$$
M_{\mathrm{SPIOs}}=\rho V
$$

where $\rho=6000 \mathrm{~kg} / \mathrm{m}^{3}$ [42]. A double-layer model can also be used to estimate SPIOs' aggregation inside the cell [30]. 


\section{Conclusions}

This in vitro study indicates that electroporation in the presence of the clinically used transfection agent, protamine sulphate (PS), can provide an effective and fast technique for labelling of various cell types with magnetic nanoparticles (e.g., ferumoxides, a clinical proved SPIOs). The Quantichrom iron assay provides a simpler method for iron determination in vitro, compared to atomic spectrometry, MR relaxometry and radioactive detection. The MFM scan provides an unique method for observing any (not just iron-based) cellular uptake of magnetic particles ranging from the nano-meter up to 100 micro-meter scale, and further quantitative information of individual cells can be derived from image processing using simple or complex models.

Electro-magnetoporation can provide an efficient tool for potential in situ clinical use by which in vivo quantification of cell/tissue iron uptake can be obtained by MR relaxometry in addition to tracking of the labelled cells by MRI.

\section{Acknowledgments}

The authors would like to express deep appreciation to tissue culture assistance from Lijun Wang and AFM assistance from Paul Prentice, Paul Campbell and Donald McLean in Campbell lab at University of Dundee. This work was supported by the Engineering and Physical Sciences Research Council (EPSRC), UK, under Grant EP/EO1514X/1 and Grant EP/HO10033/1.

\section{Conflict of Interest}

The authors declare no conflict of interest.

\section{References}

1. Thorek, D.L.; Chen, A.K.; Czupryna, J.; Tsourkas, A. Superparamagnetic iron oxide nanoparticle probes for molecular imaging. Ann. Biomed. Eng. 2006, 34, 23-38.

2. Pankhurst, Q.A.; Thanh, N.T.K.; Jones, S.K.; Dobson, J. Progress in applications of magnetic nanoparticles in biomedicine. J. Phys. D Appl. Phys. 2009, 42, doi:10.1088/0022-3727/ 42/22/224001.

3. Frimpong, R.A.; Hilt, J.Z. Magnetic nanoparticles in biomedicine: synthesis, functionalization and applications. Nanomedicine (Lond) 2010, 5, 1401-1414.

4. Pinkse, G.G.; Steenvoorde, E.; Hogendoorn, S.; Noteborn, M.; Terpstra, O.T.; Bruijn, J.A.; de Heer, E. Stable transplantation results of magnetically retracted islets: A novel method. Diabetologia 2004, 47, 55-61.

5. Alexiou, C.; Jurgons, R.; Schmid, R.J.; Bergemann, C.; Henke, J.; Erhardt, W.; Huenges, E.; Parak, F. Magnetic drug targeting-biodistribution of the magnetic carrier and the chemotherapeutic agent mitoxantrone after locoregional cancer treatment. J. Drug Target. 2003, 11, 139-149.

6. Alexiou, C.; Arnold, W.; Klein, R.J.; Parak, F.G.; Hulin, P.; Bergemann, C.; Erhardt, W.; Wagenpfeil, S.; Lubbe, A.S. Locoregional cancer treatment with magnetic drug targeting. Cancer Res. 2000, 60, 6641-6648. 
7. Ivkov, R.; DeNardo, S.J.; Daum, W.; Foreman, A.R.; Goldstein, R.C.; Nemkov, V.S.; DeNardo, G.L. Application of high amplitude alternating magnetic fields for heat induction of nanoparticles localized in cancer. Clin. Cancer Res. 2005, 11, 7093s-7103s.

8. Matuszewski, L.; Persigehl, T.; Wall, A.; Schwindt, W.; Tombach, B.; Fobker, M.; Poremba, C.; Ebert, W.; Heindel, W.; Bremer, C. Cell tagging with clinically approved iron oxides: Feasibility and effect of lipofection, particle size, and surface coating on labeling efficiency. Radiology 2005, $235,155-161$.

9. Oudkerk, M.; van den Heuvel, A.G.; Wielopolski, P.A.; Schmitz, P.I.; Borel Rinkes, I.H.; Wiggers, T. Hepatic lesions: detection with ferumoxide-enhanced T1-weighted MR imaging. Radiology 1997, 203, 449-456.

10. Zhang, B.; Jiang, B.; Chen, Y.; Huang, H.; Xie, Q.; Kang, M.; Zhang, H.; Zhai, C.; Wu, Y. Detection of viability of transplanted beta cells labeled with a novel contrast agent - polyvinylpyrrolidone-coated superparamagnetic iron oxide nanoparticles by magnetic resonance imaging. Contrast Media Mol. Imaging 2012, 7, 35-44.

11. Bulte, J.W.; Kraitchman, D.L. Iron oxide MR contrast agents for molecular and cellular imaging. NMR Biomed. 2004, 17, 484-499.

12. Sykova, E.; Jendelova, P. In vivo tracking of stem cells in brain and spinal cord injury. Prog. Brain Res. 2007, 161, 367-383.

13. Jiang, J.; Chen, Y.; Zhu, Y.; Yao, X.; Qi, J. Efficient in vitro labeling of human prostate cancer cells with superparamagnetic iron oxide nanoparticles. Cancer Biother. Radiopharm. 2011, 26, 461-467.

14. Sun, J.H.; Zhang, Y.L.; Nie, C.H.; Qian, S.P.; Yu, X.B.; Xie, H.Y.; Zhou, L.; Zheng, S.S. In vitro labeling of endothelial progenitor cells isolated from peripheral blood with superparamagnetic iron oxide nanoparticles. Mol. Med. Rep. 2012, 6, 282-286.

15. Wang, Y.X. Superparamagnetic iron oxide based MRI contrast agents: Current status of clinical application. Quant. Imaging Med. Surg. 2012, 1, 35-40.

16. Corot, C.; Robert, P.; Idee, J.-M.; Port, M. Recent advances in iron oxide nanocrystal technology for medical imaging. Adv. Drug Deliv. Rev. 2006, 58, 1471-1504.

17. Zhu, X.M.; Wang, Y.X.; Leung, K.C.; Lee, S.F.; Zhao, F.; Wang, D.W.; Lai, J.M.; Wan, C.; Cheng, C.H.; Ahuja, A.T. Enhanced cellular uptake of aminosilane-coated superparamagnetic iron oxide nanoparticles in mammalian cell lines. Int. J. Nanomed. 2012, 7, 953-964.

18. Zhang, Y.; Kohler, N.; Zhang, M. Surface modification of superparamagnetic magnetite nanoparticles and their intracellular uptake. Biomaterials 2002, 23, 1553-1561.

19. Foster, P.J.; Dunn, E.A.; Karl, K.E.; Snir, J.A.; Nycz, C.M.; Harvey, A.J.; Pettis, R.J. Cellular magnetic resonance imaging: In vivo imaging of melanoma cells in lymph nodes of mice. Neoplasia 2008, 10, 207-216.

20. Lewin, M.; Carlesso, N.; Tung, C.H.; Tang, X.W.; Cory, D.; Scadden, D.T.; Weissleder, R. Tat peptide-derivatized magnetic nanoparticles allow in vivo tracking and recovery of progenitor cells. Nat. Biotechnol. 2000, 18, 410-414.

21. Buerli, T.; Pellegrino, C.; Baer, K.; Lardi-Studler, B.; Chudotvorova, I.; Fritschy, J.M.; Medina, I.; Fuhrer, C. Efficient transfection of DNA or shRNA vectors into neurons using magnetofection. Nat. Protoc. 2007, 2, 3090-3101. 
22. Walczak, P.; Ruiz-Cabello, J.; Kedziorek, D.A.; Gilad, A.A.; Lin, S.; Barnett, B.; Qin, L.; Levitsky, H.; Bulte, J.W.M. Magnetoelectroporation: improved labeling of neural stem cells and leukocytes for cellular magnetic resonance imaging using a single FDA-approved agent. Nanomed. Nanotechnol. Biol. Med. 2006, 2, 89-94.

23. Prentice, P.; Cuschierp, A.; Dholakia, K.; Prausnitz, M.; Campbell, P. Membrane disruption by optically controlled microbubble cavitation. Nat. Phys. 2005, 1, 107-110.

24. Bulte, J.W.M.; Douglas, T.; Witwer, B.; Zhang, S.C.; Strable, E.; Lewis, B.K.; Zywicke, H.; Miller, B.; van Gelderen, P.; Moskowitz, B.M.; et al. Magnetodendrimers allow endosomal magnetic labeling and in vivo tracking of stem cells. Nat. Biotechnol. 2001, 19, 1141-1147.

25. Leung, K. Ferumoxides. Molecular Imaging and Contrast Agent Database (MICAD). Available online: http://www.ncbi.nlm.nih.gov/books/NBK23037/(accessed on 26 January 2013).

26. Frank, J.A.; Miller, B.R.; Arbab, A.S.; Zywicke, H.A.; Jordan, E.K.; Lewis, B.K.; Bryant, L.H., Jr.; Bulte, J.W. Clinically applicable labeling of mammalian and stem cells by combining superparamagnetic iron oxides and transfection agents. Radiology 2003, 228, 480-487.

27. Wang, L.; Wang, Z.; Frank, T.G.; Brown, S.I.; Chudek, S.A.; Cuschieri, A. Rapid and efficient cell labeling with a MRI contrast agent by electroporation in the presence of protamine sulfate. Nanomedicine (Lond) 2009, 4, 305-315.

28. Amemiya, Y.; Tanaka, T.; Yoza, B.; Matsunaga, T. Novel detection system for biomolecules using nano-sized bacterial magnetic particles and magnetic force microscopy. J. Biotechnol. 2005, 120, 308-314.

29. Shen, H.B.; Long, D.H.; Zhu, L.Z.; Li, X.Y.; Dong, Y.M.; Jia, N.Q.; Zhou, H.Q.; Xin, X.; Sun, Y. Magnetic force microscopy analysis of apoptosis of HL-60 cells induced by complex of antisense oligonucleotides and magnetic nanoparticles. Biophys. Chem. 2006, 122, 1-4.

30. Zhang, Y.; Yang, M.; Ozkan, M.; Ozkan, C.S. Magnetic force microscopy of iron oxide nanoparticles and their cellular uptake. Biotechnol. Prog. 2009, 25, 923-928.

31. Huberman, A.; Perez, C. Nonheme iron determination. Anal. Biochem. 2002, 307, 375-378.

32. Wu, Y.J.; Muldoon, L.L.; Varallyay, C.; Markwardt, S.; Jones, R.E.; Neuwelt, E.A. In vivo leukocyte labeling with intravenous ferumoxides/protamine sulfate complex and in vitro characterization for cellular magnetic resonance imaging. Am. J. Physiol. Cell Physiol. 2007, 293, C1698-C1708.

33. Riemer, J.; Hoepken, H.H.; Czerwinska, H.; Robinson, S.R.; Dringen, R. Colorimetric ferrozine-based assay for the quantitation of iron in cultured cells. Anal. Biochem. 2004, 331, 370-375.

34. Brekke, C.; Morgan, S.C.; Lowe, A.S.; Meade, T.J.; Price, J.; Williams, S.C.; Modo, M. The in vitro effects of a bimodal contrast agent on cellular functions and relaxometry. NMR Biomed. 2007, 20, 77-89.

35. Kamau, S.W.; Hassa, P.O.; Steitz, B.; Petri-Fink, A.; Hofmann, H.; Hofmann-Amtenbrink, M.; von Rechenberg, B.; Hottiger, M.O. Enhancement of the efficiency of non-viral gene delivery by application of pulsed magnetic field. Nucleic Acids Res. 2006, 34, e40.

36. Mykhaylyk, O.; Antequera, Y.S.; Vlaskou, D.; Plank, C. Generation of magnetic nonviral gene transfer agents and magnetofection in vitro. Nat. Protoc. 2007, 2, 2391-2411. 
37. Lee, C.H.; Kim, E.Y.; Jeon, K.; Tae, J.C.; Lee, K.S.; Kim, Y.O.; Jeong, M.Y.; Yun, C.W.; Jeong, D.K.; Cho, S.K.; et al. Simple, efficient, and reproducible gene transfection of mouse embryonic stem cells by magnetofection. Stem Cells Dev. 2008, 17, 133-141.

38. Park, H.Y.; Noh, E.H.; Chung, H.M.; Kang, M.J.; Kim, E.Y.; Park, S.P. Efficient generation of virus-free iPS cells using liposomal magnetofection. PLoS One 2012, 7, e45812.

39. Furlani, E.P.; Xue, X. A model for predicting field-directed particle transport in the magnetofection process. Pharm. Res. 2012, 29, 1366-1379.

40. Hoskins, C.; Wang, L.; Cheng, W.P.; Cuschieri, A. Dilemmas in the reliable estimation of the in vitro cell viability in magnetic nanoparticle engineering: which tests and what protocols? Nanoscale Res. Lett. 2012, 7, 77.

41. Liu, D.; Wang, L.; Wang, Z.; Cuschieri, A. Magnetoporation and magnetolysis of cancer cells via carbon nanotubes induced by rotating magnetic fields. Nano Lett. 2012, 12, 5117-5121.

42. Hong, R.; Cima, M.J.; Weissleder, R.; Josephson, L. Magnetic microparticle aggregation for viscosity determination by MR. Magn. Reson. Med. 2008, 59, 515-520.

(C) 2013 by the authors; licensee MDPI, Basel, Switzerland. This article is an open access article distributed under the terms and conditions of the Creative Commons Attribution license (http://creativecommons.org/licenses/by/3.0/). 